\title{
Ferrimagnetic Resonance Measurements Using IF Substitution Techniques
}

\author{
W. E. Case,* R. D. Harrington, and L. B. Schmidt
}

(April 24, 1964)

\begin{abstract}
Advantages of using an IF substitution method with a waveguide below cutoff attenuator for obtaining ferrimagnetic resonance line width of polycrystalline materials are discussed. An improvement in the IF system involving phase locking the local oscillator to the signal generator is described. Measurements were obtained which indicate that the above IF attenuator method compares favorably with accurate frequency measuring techniques for plotting entire resonance curves.
\end{abstract}

\section{Introduction}

The American Society for Testing and Materials has recently established a standard test method for determining the ferrimagnetic resonance line width and gyromagnetic ratio of nonmetallic magnetic materials [1]. ${ }^{1}$ The method essentially utilizes a precision $\mathrm{RF}$ attenuator located on the input side of the transmission cavity for determining the halfpower points on the resonance curve of the sample. We have recently published tables which facilitate the calculation of the half-power points using the ASTM techniques [2]. At the same time, some of the advantages and disadvantages of using an IF substitution technique with a $30 \mathrm{Mc} / \mathrm{s}$ piston attenuator for obtaining these measurements were pointed out.

A typical advantage of IF detection may be found in greater sensitivity which increases the range of sample size and loss which can be measured. Another advantage is related to the increased accuracy inherent in the use of an IF substitution method using a waveguide below cutoff attenuator [3]. Furthermore, since the IF frequency is constant in such a setup, the same attenuator and detection system may be used in equipment designed for measurements at different microwave frequencies.

The primary disadvantage associated with the IF substitution method lies in the inconvenience and additional time required for manually tracking the local IF oscillator with the signal oscillator. This introduces delay in the time needed for the measurement of the maximum and $3 \mathrm{~dB}$ points on the resonant curve of the sample since the signal generator must be retuned at these points to maintain the cavity at resonance. The IF method of course requires somewhat more equipment than the $\mathrm{RF}$ substitution technique; however, with the exception of a precision waveguide below cutoff attenuator, the extra equipment is usually available in a typical laboratory. Furthermore, at the present time,

*Radio Standards Physies Division, NBS Boulder Laboratories, Boulder, Colo

${ }_{1}$ Figures in brackets indicate the literature references at the end of this paper. complete IF attenuation measuring systems are becoming commercially available.

Our previous experience using the method adopted by the ASTM with an IF substitution arrangement had indicated that the advantages in many cases outweighed the disadvantages. For this reason, we have recently carried out further studies in an effort to gain a greater appreciation of the applicability of IF substitution methods in ferrimagnetic resonance setups in general as well as to develop a system for avoiding the disadvantage associated with local oscillator tuning. The purpose of this paper is to describe in somewhat more detail the results of these studies. This discussion will be divided into essentially two areas. First of all, the development of a ferrimagnetic resonance measurement system using a phase lock arrangment for automatically tracking the local oscillator with the signal oscillator will be described. Secondly, a comparison of the use of the attenuation method using a waveguide below cutoff attenuator with a method using an accurate frequency measuring technique for determining entire resonance loss curves will be discussed.

\section{Experimental Setup}

A typical experimental setup utilizing an IF substitution technique designed for measuring line width by means of the ASTM method has a waveguide below cutoff attenuator preceding the IF detector. Changes in the RF attenuation of the cavity due to the sample properties are measured by the corresponding changes in the IF attenuator which maintain a constant level signal to the IF detector. In a typical measurement of line width, the signal frequency is first adjusted to give cavity resonance for the empty cavity and the IF attenuator is set to a value, $A_{0}$, to give a convenient output reference level on the galvanometer. The sample is then inserted, the d-c magnetic field adjusted for maximum absorption in the sample, the frequency adjusted for cavity resonance, and the IF attenuator set to a new value which we designate as $A_{r}$ in order to obtain the 
same output reference level. Knowing $A_{0}-A_{r}$, it is possible to calculate the IF attenuator reading corresponding to the half-power points on the resonance curve of the sample. The attenuator is then set at this calculated value, and the line width is determined from the two d-c field settings necessary to alter the sample properties until the fixed output reference signal is obtained. Once again, the signal frequency must be adjusted to maintain cavity resonance corresponding to these new sample conditions. It is readily apparent from the above discussion that the magnetic d-c field and signal oscillator frequency settings are interdependent and require considerable trail and error adjustment for obtaining optimum conditions. The added complication of tuning a local oscillator to maintain a fixed IF frequency is obvious.

The availability of commercial phase lock systems offers a solution to the above problem. Such systems are usually used to lock the frequency of a klystron oscillator to some multiple of a low frequency crystal oscillator to produce stable microwave frequencies. The same equipment should be applicable for phase locking the klystron local oscillator to the signal oscillator in an IF system. An arrangement using a frequency discriminator to vary the local oscillator frequency to automatically track the frequency variation of the $\mathrm{RF}$ source has already been described in the literature [4].

We have recently developed a ferrimagnetic resonance measurement setup using a commercially available phase lock system in a $30 \mathrm{Mc} / \mathrm{s}$ IF substitution arrangement. In this system, the local oscillator is phase locked to a frequency $30 \mathrm{Mc} / \mathrm{s}$ below the signal frequency. A block diagram of this setup designed for a signal frequency, $f_{0}$, is shown in figure 1 . Basically, the phase lock system compares the difference of the signal frequency $f_{0}$ and the IF local oscillator frequency $f_{0}-30 \mathrm{Mc} / \mathrm{s}$ with a $30 \mathrm{Mc} / \mathrm{s}$ reference signal which is contained in the phase-lock unit. A d-c correction signal is applied to the local oscillator, a klystron, such that it tracks the signal oscillator. This local oscillator signal at a frequency of $f_{0}-30 \mathrm{Mc} / \mathrm{s}$ is, of course, mixed with the cavity output signal at the frequency $f_{0}$ to produce

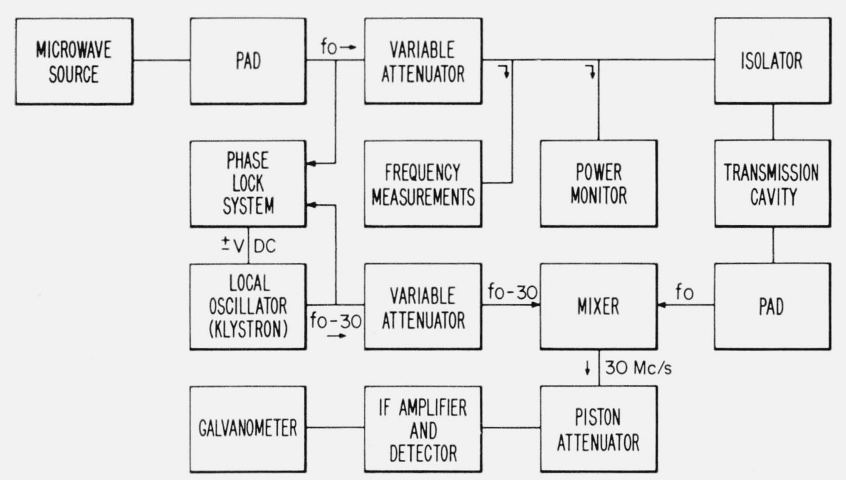

Figure 1. Schematic diagram of equipment used for ferrimagnetic resonance work using a phase-locked IF substitution system. the IF frequency of $30 \mathrm{Mc} / \mathrm{s}$. All attenuation measurements are made using the $30 \mathrm{Mc} / \mathrm{s}$ piston attenuator which controls the level of this IF signal. The remaining components in the system are used for level setting, power monitoring, and frequency measurements which are all common to setups of this type. These measurements also require $\mathrm{d}-\mathrm{c}$ field measuring equipment such as a nuclear resonance gaussmeter, which is not shown in the diagram.

It should be recognized that attenuation measurements such as above require that the power levels in the overall system remain stable during a measurement. This is, of course, true for both the RF and IF substitution methods. A good temperature independent power monitoring setup is thus necessary in order that corrections can be made for any small variations in the power level of the system which may occur during a measurement. The crystal used in the power monitoring unit shown in figure 1 was mounted in a temperature controlled oven.

It is also apparent that the accuracy of measurements of this type is dependent upon the type of precision attenuator used. The piston attenuator used in the setup in figure 1 can detect attenuation changes of $0.01 \mathrm{~dB}$. This type of performance is considerably better than can be obtained from most RF attenuators. Furthermore, accurate RF attenuators are in general available only over limited microwave frequency ranges. This frequency restriction is not applicable to the piston attenuator operating at the fixed IF frequency. Impedance matching is much less of a problem in piston attenuators than is the case for RF attenuators. While it is recognized that the insertion loss of a piston attenuator is rather high, this is not a serious disadvantage due to the high sensitivity in the IF detection system and the fact that the range of attenuation of concern in an ordinary measurement is not great.

An experimental evaluation of the above setup has given very satisfactory results. The local oscillator accurately tracks the signal oscillator during a typical measurement. As a result, the ease of operation of the IF method with this phase lock system compares favorably with the RF substitution method ordinarily used in the ASTM method.

\section{Resonance Curve Plotting}

The discussion thus far in the paper has referred primarily to the measurement of the $3 \mathrm{~dB}$ points on the resonance curve of a sample. However, many situations arise in which a knowledge of the shape of the complete loss curve is desirable. This is usually expressed as the imaginary susceptibility $\chi^{\prime \prime}$ as a function of the applied field. Many techniques for plotting curves of this type are based on VSWR measurements using reflection type cavities [5]. Some of the earlier work in obtaining these measurements with transmission type cavities was based on the RF substitution method [6]. A more 
recent description of the use of $\mathrm{RF}$ attenuators in such work has also been given by Silber [7]. However, with the development of transfer oscillators in conjunction with frequency counters, it has become possible to more accurately measure resonance curves in terms of changes in the $Q$ when transmission cavities are used. On the other hand, a study of the working equations for plotting resonance curves suggests that the use of an accurate piston attenuator such as described above should allow such curves to be obtained from IF attenuation measurements with accuracies comparable to those obtained from frequency measurements. The advantages of the $I F$ substitution method have also been recognized in the measurements of other types of materials such as high loss liquids [8]. It was thus the purpose of the present work to make a direct comparison of ferrimagnetic resonance curves obtained using a frequency counter and transfer oscillator with those obtained using a piston attenuator. In both cases, the IF system shown in figure 1 was used for obtaining the data.

It may be shown using an analysis such as described in reference 5 or 6 that the effective imaginary susceptibility of a small sample in a transmission cavity is given by

$$
\chi^{\prime \prime}=\frac{1}{2}\left(\frac{1}{Q_{n}}-\frac{1}{Q_{0}}\right) C \frac{V_{c}}{V_{s}}
$$

where

$Q_{n}=$ loaded $Q$ of the cavity with the sample;

$Q_{0}=$ loaded $Q$ of the empty cavity;

$C=$ a constant dependent on the cavity and its mode;

$V_{c}, V_{s}=$ volume of cavity and sample respectively.

When using the frequency method, the $Q$ 's in this equation are evaluated from $f / \Delta f$, where $f$ is the resonant frequency and $\Delta f$ is the frequency difference between the three $\mathrm{dB}$ points on the resonance curve. It might be mentioned that even for this frequency method, the accuracy of the piston attenuator is useful in calibrating these half-power points on the cavity resonance curve.

When using the attenuation method with a transmission cavity, it may be shown that eq (1) [see appendix] becomes

$$
\chi^{\prime \prime}=\frac{1}{2 Q_{0}}\left(10^{\frac{A_{0}-A_{n}}{20}}-1\right) C \frac{V_{c}}{V_{s}},
$$

where

$A_{0}=$ piston attenuator reading corresponding to empty cavity at resonance;

$A_{n}=$ piston attenuator reading corresponding to any point on resonance curve of sample with cavity at resonance.

Measurements were taken on several different materials which directly compared the use of the frequency counter and eq (1) with the use of the piston attenuator and eq (2). Typical data taken at 110 $\mathrm{Mc} / \mathrm{s}$ on one of these ferrite materials is shown in figure 2. It was found that excellent agreement was obtained between the two methods for several different materials provided care was taken to avoid or compensate for drift in the gain of the IF detection system in the attenuation measurement. As was pointed out for the RF substitution measurements described in the literature $[6,7]$, this drift may readily be corrected by using a high field reference level corresponding to the empty cavity.

It is felt that one of the more serious errors in either the frequency or attenuation method would be due to the dependence of the transmission loss of various components in the system on frequency. Both methods of course require small frequency changes to maintain the cavity at resonance during a measurement. Such effects are difficult to evaluate for both methods; however, errors due to the frequency sensitivity of transmission loss, impedance mismatches, etc., should be slight if only small frequency variations are encountered. Data obtained on disks such as shown in figure 2 indicated that the frequency and attenuation method in general agreed to better than 1 to 2 percent in the vicinity of the half-power points on the curves where the greatest frequency changes were necessary to maintain resonance in the cavity. This agreement suggests that transmission errors such as described above are in general quite small. Somewhat larger variations between the two methods are experienced in the wings of the resonance curve; however, the losses
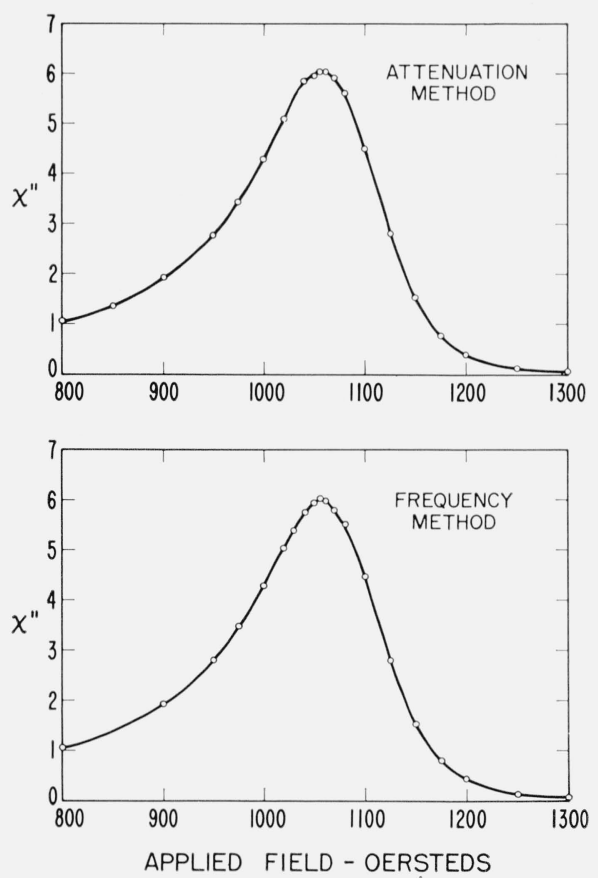

Figure 2. Typical ferrimagnetic resonance loss curve of a thin disk sample at $1100 \mathrm{Mc} / \mathrm{s}$ obtained from frequency and attenuation measurements. 
are becoming so low in these areas that accurate measurements by either method becomes difficult.

The curves shown in figure 2 give the imaginary susceptibility of the material in the vicinity of ferrimagnetic resonance as a function of $d-c$ field. In order to obtain the attenuation curve in terms of susceptibility, it was necessary to obtain a measurement of the loaded $Q$ of the empty cavity as can be seen in eq (2). This would in general be obtained with the frequency method. However, this $Q$ is assumed constant during the measurement and thus eq (2) can be written

$$
\chi^{\prime \prime}=K\left(10^{\frac{A_{0}-A_{n}}{20}}-1\right)
$$

where

$$
K=\frac{1}{2 Q_{0}} C \frac{V_{c}}{V_{s}}
$$

If $A_{n}=A_{r}$ and $\chi^{\prime \prime}=\chi^{\prime \prime}$ at the maximum point on the resonance curve, then

$$
\chi_{0}^{\prime \prime}=K\left(10^{\frac{i 0_{0}-A_{r}}{20}}-1\right)
$$

and

$$
\frac{\chi^{\prime \prime}}{\chi_{0}^{\prime \prime}}=\frac{10^{\frac{A_{0}-A_{n}}{20}}-1}{10^{\frac{A_{0}-A_{\tau}}{20}}-1},
$$

which essentially means that the shape of the resonance curve normalized to its maximum value may be obtained by attenuation measurements alone. This may be convenient in various research programs.

\section{Summary}

It is concluded that the IF substitution technique may be applied to the ASTM method of line width measurement. It has particular advantages when measuring high loss samples or when data is desired at microwave frequencies where good RF attenuators are difficult to obtain. The convenience of the IF substitution method is considerably improved by phase locking the local IF oscillator to the signal oscillator. It has been demonstrated that this system can be used to plot entire resonance curves in terms of attenuation measurements with an accuracy comparable to that obtained with a frequency counter.

\section{Appendix}

Starting with eq (1),

$$
\chi^{\prime \prime}=\frac{1}{2}\left(\frac{1}{Q_{n}}-\frac{1}{Q_{0}}\right) C \frac{V_{c}}{V_{s}},
$$

it can be shown from eq (9), p. 291, of reference [9] that

$$
\frac{P_{0}}{P_{i}}=\frac{4 Q_{0}^{2}}{Q_{1} Q_{2}}
$$

for the empty cavity and

$$
\frac{P_{n}}{P_{i}}=\frac{4 Q_{n}^{2}}{Q_{1} Q_{2}}
$$

for the cavity with sample where:

$P_{0}$ is the power output from the empty cavity;

$P_{i}$ is the power input to the cavity;

$P_{n}$ is the power output from the cavity with sample;

$Q_{0}$ is loaded $Q$ of empty cavity;

$Q_{n}$ is loaded $Q$ of cavity with sample;

$Q_{1}$ and $Q_{2}$ are the coupling $Q$ 's of the input and output ports and are assumed constant during the measurements.

If $P_{i}$ is held constant as is the case for the IF system,

$$
\frac{P_{0}}{P_{n}}=\left(\frac{Q_{0}}{Q_{n}}\right)^{2},
$$

and substituting in equation (1)

$$
\chi^{\prime \prime}=\frac{1}{2 Q_{0}}\left(\sqrt{\frac{P_{0}}{P_{n}}}-1\right) C \frac{V_{c}}{V_{s}} .
$$

This same equation is also given in references [6] and [7] except the power ratio is expressed in terms of equivalent voltage ratios.

Let the power input to the piston attenuator be $B P_{0}$ for the empty cavity and $B P_{n}$ for the cavity with the sample where $B$ is a proportionality constant. If $P$ is the constant output power level of the piston attenuator, then the corresponding attenuator readings in decibels are given by

$$
A_{0}=10 \log _{10} \frac{B P_{0}}{P}
$$

and

$$
\begin{aligned}
A_{n} & =10 \log _{10} \frac{B P_{n}}{P}, \\
A_{0}-A_{n} & =10 \log _{10} \frac{P_{0}}{P_{n}}=20 \log _{10} \frac{\sqrt{P_{0}}}{\sqrt{P_{n}}} \\
\frac{\sqrt{P_{0}}}{\sqrt{P_{n}}} & =10^{\frac{A_{0}-A_{n}}{20}} ;
\end{aligned}
$$

therefore,

$$
\chi^{\prime \prime}=\frac{1}{2 Q_{0}}\left(10^{\frac{A_{0}-A_{n}}{29}}-1\right) C \frac{V_{c}}{V_{s}},
$$

which is eq (2) in the text. 


\section{References}

[1] ASTM Test Method C524-63T. This method is under the jurisdiction of the ASTM Committee C-25 on Ceramics for Electronics.

[2] C. C. Preston and W. E. Case, Tables to facilitate the determination of the ferrimagnetic resonance line width of nonmetallic magnetic materials, NBS Tech. Note No. 173 (April 1963).

[3] R. E. Grantham and J. J. Freeman, A standard of attenuation for microwave measurements, AIEE Trans. 6\%, 329-335 (1948).

[4] B. O. Weinschel, An accurate attenuation measuring system with great dynamic range, Microwave Jour. pp. 7783 (September 1961).
15] J. O. Artman and P. E. Tannenwald, Measurement of susceptibility in ferrites, J. Appl. Phys. 26, No. 9, 1124-1132 (1955).

[6] E. G. Spencer, R. C. LeCraw, and F. Reggia, Measurement of microwave dielectric constants and tensor permeabilities of ferrite spheres, Proc. IRE 44, 790800 (1956).

[7] Handbook of microwave measurements, edited by M. Sucher and J. Fox, Polytechnic Institute of Brooklyn, Microwave Research Institute, Brooklyn, New York pp. $777-803$ (1963).

[8] J. A. Lane and J. A. Saxton, Dielectric dispersion in pure polar liquids at very high radio-frequencies, Royal Soc. of London Proc. Ser. A, 213, p. 400 (1952).

[9] C. G. Montgomery (ed.), Techniques of microwave measurements, pp. 289-291 (McGraw-Hill Book Co., Inc., New York, N.Y., 1947).

(Paper 68C4-172 